\title{
The symmetry in the martingale inequality
}

\author{
Sungchul Lee ${ }^{\mathrm{a}, *, 1}$, Zhonggen $\mathrm{Su}^{\mathrm{a}, \mathrm{b}, 2}$ \\ ${ }^{a}$ Department of Mathematics, Yonsei University, Seoul 120-749, South Korea \\ ${ }^{\mathrm{b}}$ Department of Mathematics, Zhejiang University, Hangzhou 310028, People's Republic of China
}

Received April 2001; received in revised form August 2001

\begin{abstract}
In this paper, we establish a martingale inequality and develop the symmetry argument to use this martingale inequality. We apply this to the length of the longest increasing subsequences and the independence number of sparse random graphs. (c) 2002 Elsevier Science B.V. All rights reserved.
\end{abstract}

MSC: primary 60D05; 60F05; secondary 60K35; 05C05; 90C27

Keywords: Bounded martingale inequality; Longest increasing subsequence; Independence number

\section{Introduction and main results}

A common feature in many probabilistic arguments is to show that with high probability a random variable is concentrated on its mean. The usual way to do this is via either the martingale inequality, the isoperimetric inequality, or the log-Sobolev inequality. See Godbole and Hitczenko (1998), Janson et al. (2000), McDiarmid (1997, 1989), Steele (1997), Talagrand (1995) and Vu (2001) for various extensions and beautiful applications. In this paper, we establish a martingale inequality and develop the symmetry argument to use this martingale inequality. We apply this to the length of the longest increasing subsequences and the independence number of sparse random graphs.

To motivate the discussion below, let us begin with the well-known Azuma's inequality. Given a probability space $(\Omega, \mathscr{F}, P)$ and a filtration $\mathscr{F}_{0}=\{\emptyset, \Omega\} \subset \mathscr{F}_{1} \subset \cdots \subset \mathscr{F}_{n}=\mathscr{F}$, an integrable random

\footnotetext{
${ }^{*}$ Corresponding author.

E-mail addresses: sungchul@yonsei.ac.kr (S. Lee), zgsu@mail.hz.zj.cn, zgsu2001@yahoo.com (Z. Su).

${ }^{1}$ This work was supported by the BK21 project of the Department of Mathematics, Yonsei University, the interdisciplinary research program of KOSEF 1999-2-103-001-5, and $\mathrm{Com}^{2} \mathrm{MaC}$ in POSTECH.

${ }^{2}$ This work was supported by the BK21 project of the Department of Mathematics, Yonsei University, and by NSFC 10071072.
} 
variable $X \in L^{1}(\Omega, \mathscr{F}, P)$ can be written as

$$
X-E X=\sum_{k=1}^{n} E\left(X \mid \mathscr{F}_{k}\right)-E\left(X \mid \mathscr{F}_{k-1}\right):=\sum_{k=1}^{n} d_{k} .
$$

Here $d_{k}$ is a martingale difference. If there exist constants $c_{k}>0$ such that $\left|d_{k}\right| \leqslant c_{k}$ a.s. for each $k \leqslant n$, then for every $t \geqslant 0$,

$$
\begin{aligned}
& P(X \geqslant E X+t) \leqslant \exp \left(-\frac{t^{2}}{2 \sum_{k=1}^{n} c_{k}^{2}}\right), \\
& P(X \leqslant E X-t) \leqslant \exp \left(-\frac{t^{2}}{2 \sum_{k=1}^{n} c_{k}^{2}}\right) .
\end{aligned}
$$

The above result appears in Azuma (1967) and is often called Azuma's inequality, although it was actually earlier given by Hoeffding (1963). In most applications, $X$ is a function of $n$-independent (possibly vector valued) random variables $\xi_{1}, \xi_{2}, \ldots, \xi_{n}$ and the filtration is

$$
\mathscr{F}_{k}=\sigma\left(\xi_{1}, \xi_{2}, \ldots, \xi_{k}\right)
$$

In this case, we let $\left\{\xi_{1}^{\prime}, \xi_{2}^{\prime}, \ldots, \xi_{n}^{\prime}\right\}$ be an independent copy of $\left\{\xi_{1}, \xi_{2}, \ldots, \xi_{n}\right\}$ and define

$$
\begin{aligned}
& \Delta_{k}=X\left(\xi_{1}, \ldots, \xi_{k-1}, \xi_{k}, \xi_{k+1}^{\prime}, \ldots, \xi_{n}^{\prime}\right)-X\left(\xi_{1}, \ldots, \xi_{k-1}, \xi_{k}^{\prime}, \xi_{k+1}^{\prime}, \ldots, \xi_{n}^{\prime}\right), \\
& d_{k}=E\left(\Delta_{k} \mid \mathscr{F}_{k}\right) .
\end{aligned}
$$

By definition, $\Delta_{k}$ is the change in the value of $X$ resulting from a change only in one coordinate. So, if $\left|\Delta_{k}\right| \leqslant c_{k}$ a.s., then $\left|d_{k}\right| \leqslant c_{k}$ a.s. and we can apply Azuma's inequality to obtain a tail bound for $X$. However, in many cases $c_{k}$ grows too rapidly that Azuma's inequality does not provide any reasonable tail bound. A detailed analysis on various problems in our paper shows that our $\Delta_{k}$ 's are much smaller than $c_{k}$ most of the time and from this observation we can improve Azuma's inequality and obtain a reasonable tail bound for $X$. Our result is the following.

Theorem 1. Let $X$ be an integrable random variable defined on a probability space $(\Omega, \mathscr{F}, P)$ which is in fact a function of $n$-independent random variables $\xi_{1}, \xi_{2}, \ldots, \xi_{n}$. We define $\mathscr{F}_{k}, \Delta_{k}, d_{k}$ by (1.1)-(1.3). Assume that there exists a positive and finite constant $c$ such that for all $k \leqslant n$

$$
\left|\Delta_{k}\right| \leqslant c \text { a.s. }
$$

and there exist $0<p_{k}<1$ such that for each $k \leqslant n$

$$
P\left(0<\left|\Delta_{k}\right| \leqslant c \mid \widetilde{F}_{k-1}\right) \leqslant p_{k} \text { a.s. }
$$

Then, for every $t>0$

$$
\begin{aligned}
& P(X \geqslant E X+t) \leqslant \exp \left(-\frac{t^{2}}{2 c^{2} \sum_{k=1}^{n} p_{k}+2 c t / 3}\right), \\
& P(X \leqslant E X-t) \leqslant \exp \left(-\frac{t^{2}}{2 c^{2} \sum_{k=1}^{n} p_{k}+2 c t / 3}\right) .
\end{aligned}
$$


Proof. We prove here (1.6) with $c=1$. From this one can easily get (1.6). The argument for (1.7) is the same. Let $\bar{p}=n^{-1} \sum_{k=1}^{n} p_{k}$ and let $g(x)=\left(\mathrm{e}^{x}-1-x\right) / x^{2}$ with $g(0)=\frac{1}{2}$. Since $d_{k}=E\left(\Delta_{k} \mid \mathscr{F}_{k-1}\right)$, by Jensen's inequality we have for any $s>0$

$$
\begin{aligned}
E\left(\mathrm{e}^{s d_{k}} \mid \mathscr{F}_{k-1}\right) & =E\left(\mathrm{e}^{\left.s E\left(\Delta_{k} \mid \mathscr{F}_{k-1}\right) \mid \mathscr{F}_{k-1}\right)}\right. \\
& \leqslant E\left(\mathrm{e}^{s \Delta_{k}} \mid \mathscr{F}_{k-1}\right) \\
& =E\left(1+s \Delta_{k}+s^{2} \Delta_{k}^{2} g\left(s \Delta_{k}\right) \mid \mathscr{F}_{k-1}\right) .
\end{aligned}
$$

Since $g$ is increasing and since $\left|\Delta_{k}\right| \leqslant 1$ a.s., by (1.5) we have

$$
\begin{aligned}
E\left(\mathrm{e}^{\left.s d_{k} \mid \mathscr{F}_{k-1}\right)}\right. & \leqslant E\left(1+s \Delta_{k}+s^{2} \Delta_{k}^{2} g\left(s \Delta_{k}\right) \mid \mathscr{F}_{k-1}\right) \\
& \leqslant 1+s^{2} g(s) E\left(\Delta_{k}^{2} \mid \mathscr{F}_{k-1}\right) \\
& \leqslant 1+s^{2} g(s) p_{k} \\
& \leqslant \mathrm{e}^{s^{2} g(s) p_{k}} \text { a.s. }
\end{aligned}
$$

By Markov's inequality, then we have for any $s>0$

$$
\begin{aligned}
P(X \geqslant E X+t) & \leqslant \mathrm{e}^{-s t} E \mathrm{e}^{s(X-E X)} \\
& \leqslant \mathrm{e}^{-s t} E \mathrm{e}^{s \sum_{k=1}^{n} d_{k}} \\
& \leqslant \mathrm{e}^{-s t} E \mathrm{e}^{s \sum_{k=1}^{n-1} d_{k}} E\left(\mathrm{e}^{s d_{n}} \mid \mathscr{F}_{n-1}\right) \\
& \leqslant \mathrm{e}^{-s t} E \mathrm{e}^{s \sum_{k=1}^{n-1} d_{k}} \mathrm{e}^{s^{2} g(s) p_{n}} \\
& \leqslant \cdots \\
& \leqslant \mathrm{e}^{-s t} \mathrm{e}^{s^{2} g(s) \sum_{k=1}^{n} p_{k}} \\
& =\mathrm{e}^{-s t+\left(\mathrm{e}^{s}-1-s\right) n \bar{p}} .
\end{aligned}
$$

Letting $\phi(x)=(1+x) \log (1+x)-x$ we note that $\phi(x) \geqslant x^{2} /(2(1+x / 3))$. With the choice of $s=\log (1+t / n \bar{p})$ we have then

$$
\begin{aligned}
P(X \geqslant E X+t) & \leqslant \exp (-n \bar{p} \phi(t / n \bar{p})) \\
& \leqslant \exp \left(-\frac{t^{2}}{2 \sum_{k=1}^{n} p_{k}+2 t / 3}\right) .
\end{aligned}
$$

In this paper we show a way, we call a symmetry argument, to use this inequality to obtain a reasonably good tail bound for $X$. More precisely, we show how to estimate the term $\sum_{k=1}^{n} p_{k}$ in Theorem 1. In some cases this is easy. See Luczak and McDiarmid (2001) for an example. However, in some other cases this is not an easy job at all. Actually, in his very beautiful survey McDiarmid (1997) introduced Talagrand's isoperimetric inequality by showing that the isoperimetric inequality gives a reasonable tail bound for the longest increasing subsequence whereas the martingale inequality 
does not. We were suspicious about this and this was one of our motivations of this research. What we find in this paper is that by the symmetry argument we can control the term $\sum_{k=1}^{n} p_{k}$ effectively and surprisingly by the above martingale inequality we can provide a comparable tail bound for the longest increasing subsequence. In Section 2, we tell this story. In Section 3, we again use the martingale inequality and the symmetry, and provide a tail bound for the independence number. Compared to the isoperimetric inequality and the log-Sobolev inequality, the martingale inequality is rather elementary. Therefore, if one knows the symmetry argument in this paper, in many cases one can use the elementary martingale inequality to obtain a reasonably good tail bound.

\section{The longest increasing subsequence}

Consider the symmetric group $S_{n}$ of permutations $\pi$ on the numbers $1,2, \ldots, n$, equipped with the uniform probability measure. Given a permutation $\pi=(\pi(1), \pi(2), \ldots, \pi(n))$, an increasing subsequence $i_{1}, i_{2}, \ldots, i_{k}$ is a subsequence of $1,2, \ldots, n$ such that

$$
i_{1}<i_{2}<\cdots<i_{k}, \quad \pi\left(i_{1}\right)<\pi\left(i_{2}\right)<\cdots<\pi\left(i_{n}\right) .
$$

We write $L_{n}(\pi)$ for the length of the longest increasing subsequences of $\pi$. It turns out that $L_{n}$ provides an entry to a rich and diverse circle of mathematical ideas. The recent surveys Aldous and Diaconis (1999), Deift (2000) are useful references for the properties of $L_{n}$, various associated results and some of the history. We will present below only what we need for the proof of Theorem 2.

Let $U_{i}=\left(X_{i}, Y_{i}\right), i=1,2, \ldots, n$, be a sequence of iid uniform sample on the unit square $[0,1]^{2}$. $U_{i_{1}}, U_{i_{2}}, \ldots, U_{i_{k}}$ is called a monotone increasing chain of height $k$ if

$$
X_{i_{j}}<X_{i_{j+1}}, Y_{i_{j}}<Y_{i_{j+1}} \quad \text { for } j=1,2, \ldots, k-1 .
$$

Note that by definition we do not require $i_{j}<i_{j+1}$ and hence $U_{i_{1}}, U_{i_{2}}, \ldots, U_{i_{k}}$ is in general not a subsequence of $U_{1}, U_{2}, \ldots, U_{n}$. Define $L_{n}(U)$ to be the maximum height of the chains in the sample $U_{1}, U_{2}, \ldots, U_{n}$.

A key observation, due to Hammersley (1972), is that $L_{n}(\pi)$ has the same distribution as $L_{n}(U)$. In fact, based on this equivalent formulation Hammersley (1972) first proved that there is a constant $c_{2}$ such that

$$
\frac{L_{n}(\pi)}{\sqrt{n}} \rightarrow c_{2} \text { in probability and in mean. }
$$

The constant $c_{2}$ is now known to be 2. See Aldous and Diaconis (1999) for details.

Our main result regarding the longest increasing subsequence is as follows.

Theorem 2. Given any $\varepsilon>0$, for all sufficiently large $n$ and any $t>0$

$$
P\left(\left|L_{n}(\pi)-E L_{n}(\pi)\right| \geqslant t\right) \leqslant 2 \exp \left(-\frac{t^{2}}{(16+\varepsilon) \sqrt{n}+2 t / 3}\right) .
$$


Remark. Talagrand (1995) first obtained as an application of his isoperimetric inequality that for all $t>0$,

$$
P\left(L_{n} \geqslant M_{n}+t\right) \leqslant 2 \exp \left(-\frac{t^{2}}{4\left(M_{n}+t\right)}\right), \quad P\left(L_{n} \leqslant M_{n}-t\right) \leqslant 2 \exp \left(-\frac{t^{2}}{4 M_{n}}\right) .
$$

where $M_{n}$ denotes the median of $L_{n}$. Recently, Boucheron et al. (2000) used the log-Sobolev inequality to improve the Talagrand constants as follows:

$$
P\left(L_{n} \geqslant E L_{n}+t\right) \leqslant \exp \left(-\frac{t^{2}}{2 E L_{n}+2 t / 3}\right), \quad P\left(L_{n} \leqslant E L_{n}-t\right) \leqslant \exp \left(-\frac{t^{2}}{2 E L_{n}}\right) .
$$

Comparing (2.1) with (2.2) and (2.3), and noting $M_{n} / \sqrt{n} \rightarrow 2$ as $n \rightarrow \infty$, our elementary martingale argument provides the same order of bounds for $L_{n}$.

We also remark that Baik et al. (1999) proved that $\operatorname{Var} L_{n} \approx n^{1 / 3}$ and $\left(L_{n}-2 \sqrt{n}\right) / n^{1 / 6}$ converges in distribution.

Proof. By Hammersley's equivalent formulation it suffices to show that the theorem holds for $L_{n}(U)$ instead of $L_{n}(\pi)$. Let $\left\{U_{1}^{\prime}, U_{2}^{\prime}, \ldots, U_{n}^{\prime}\right\}$ be an independent copy of $\left\{U_{1}, U_{2}, \ldots, U_{n}\right\}$. It is easy to see that, letting

$$
\Delta_{k}=L_{n}\left(U_{1}, \ldots, U_{k-1}, U_{k}, U_{k+1}^{\prime}, \ldots, U_{n}^{\prime}\right)-L_{n}\left(U_{1}, \ldots, U_{k-1}, U_{k}^{\prime}, U_{k+1}^{\prime}, \ldots, U_{n}^{\prime}\right),
$$

$\Delta_{k}$ takes values only $+1,0$, and -1 . Moreover, since $E\left(\Delta_{k} \mid \mathscr{F}_{k-1}\right)=0$ where $\mathscr{F}_{k-1}=\sigma\left(U_{1}, U_{2}, \ldots\right.$, $\left.U_{k-1}\right)$, we have

$$
P\left(\Delta_{k}=+1 \mid \mathscr{F}_{k-1}\right)=P\left(\Delta_{k}=-1 \mid \mathscr{F}_{k-1}\right) .
$$

Denote by $A_{j}$ the event that any of the highest chains of $\left(U_{1}, \ldots, U_{n}\right)$ contains $U_{j}$. Since we are only concerned with the relative position of $U_{j}$ in the $n$ sample points instead of the order, by symmetry each $A_{j}$ occurs with equal probability. Similarly, given $U_{1}, U_{2}, \ldots, U_{k-1}$, each $A_{j}$ under the point configuration $U_{1}, \ldots, U_{k-1}, U_{k}, U_{k+1}^{\prime}, \ldots, U_{n}^{\prime}$ occurs with equal probability for $j=k, k+1, \ldots, n$. Therefore, since $\Delta_{k}=+1$ implies that $A_{k}$ happens, we have

$$
\begin{aligned}
P\left(\Delta_{k}=+1 \mid \mathscr{F}_{k-1}\right) & \leqslant P\left(A_{k} \mid \mathscr{F}_{k-1}\right) \\
& =\frac{1}{n-k+1} \sum_{j=k}^{n} P\left(A_{j} \mid \mathscr{F}_{k-1}\right) \\
& =\frac{1}{n-k+1} E\left(\sum_{j=k}^{n} 1\left(A_{j}\right) \mid \mathscr{F}_{k-1}\right) .
\end{aligned}
$$

Note that $\sum_{j=k}^{n} 1\left(A_{j}\right)$ is just the number of points of $\left(U_{k}, U_{k+1}^{\prime}, \ldots, U_{n}^{\prime}\right)$, which are common to any highest chains of $\left(U_{1}, \ldots, U_{k-1}, U_{k}, U_{k+1}^{\prime}, \ldots, U_{n}^{\prime}\right)$ and hence if we collect the points $U_{j}$ or $U_{j}^{\prime}$ with $1\left(A_{j}\right)=1$, this itself forms a chain. Therefore, by the definition of chain we have

$$
E\left(\sum_{j=k}^{n} 1\left(A_{j}\right) \mid \mathscr{F}_{k-1}\right) \leqslant E L_{n-k+1}\left(U_{k}, U_{k+1}, \ldots, U_{n}\right) \text { a.s. }
$$


Letting $p_{k}=2 E L_{n-k+1}\left(U_{k}, U_{k+1}, \ldots, U_{n}\right) /(n-k+1)$, we have

$$
\begin{aligned}
P\left(\Delta_{k}=+1 \mid \mathscr{F}_{k-1}\right) & \leqslant \frac{1}{n-k+1} E\left(\sum_{j=k}^{n} 1\left(A_{j}\right) \mid \mathscr{F}_{k-1}\right) \\
& \leqslant \frac{1}{n-k+1} E L_{n-k+1}\left(U_{k}, U_{k+1}, \ldots, U_{n}\right) \\
& =\frac{p_{k}}{2} .
\end{aligned}
$$

Now we apply Theorem 1 to obtain

$$
P\left(\left|L_{n}(U)-E L_{n}(U)\right| \geqslant t\right) \leqslant 2 \exp \left(-\frac{t^{2}}{4 \sum_{k=1}^{n} E L_{k}(U) / k+2 t / 3}\right) .
$$

Since $E L_{n}(U) / \sqrt{n} \rightarrow 2$ as $n \rightarrow \infty, n^{-1 / 2} \sum_{k=1}^{n} E L_{k}(U) / k \rightarrow 4$ and hence the theorem follows.

Next let us turn to the case of $d$-dimensional chains. Let $U_{i}=\left(X_{i}^{1}, X_{i}^{2}, \ldots, X_{i}^{d}\right), i=1,2, \ldots, n$, be a sequence of iid uniform sample on the unit cube $[0,1]^{d} . U_{i_{1}}, U_{i_{2}}, \ldots, U_{i_{k}}$ is a monotone increasing chain of height $k$ if

$$
X_{i_{j}}^{1}<X_{i_{j+1}}^{1}, X_{i_{j}}^{2}<X_{i_{j+1}}^{2}, \ldots, X_{i_{j}}^{d}<X_{i_{j+1}}^{d} \quad \text { for } j=1,2, \ldots, k-1
$$

Define $L_{n, d}(U)$ to be the maximum height of the chains in the sample $U_{1}, U_{2}, \ldots, U_{n}$. By an elementary calculation Bollobás and Winkler (1988) proved that there is a constant $c_{d}$ such that

$$
\frac{L_{n, d}(U)}{n^{1 / d}} \rightarrow c_{d} \text { in probability and in mean }
$$

The exact values $c_{d}$ for $d>2$ are not known. However, there is a conjecture on $c_{d} ; c_{d}=\sum_{k=0}^{d-1} 1 / k !$. See Steele (1995) for details. With ingenuity and endeavor, Bollobás and Brightwell (1992) investigated the speed of convergence of $E L_{n, d}(U) / n^{1 / d}$ to $c_{d}$ and the concentration of $L_{n, d}(U)$ about its expectation by using the martingale inequality. Here is a better concentration bound. We skip its proof which is the same as that of Theorem 2 .

Theorem 3. Given any $\varepsilon>0$, for all sufficiently large $n$ and any $t>0$

$$
P\left(\left|L_{n, d}(U)-E L_{n, d}(U)\right| \geqslant t\right) \leqslant 2 \exp \left(-\frac{t^{2}}{4\left(d c_{d}+\varepsilon\right) n^{1 / d}+2 t / 3}\right) .
$$

\section{The independence number}

Given a complete graph $K_{n}$ and $0<p<1$, we define a random graph $G(n, p)$ by selecting each edge in $K_{n}$ with probability $p$ independently. More specifically, first we name the vertices in $K_{n}$ by $k, 1 \leqslant k \leqslant n$. Let $U_{i j}, 1 \leqslant i<j \leqslant m, m=\left(\begin{array}{c}n \\ 2\end{array}\right)$, be the independent uniform weight assigned to the edge between $i$ and $j$. Then, we keep the edges with $U \leqslant p$ and remove the edges with $U>p$. A subset $\mathscr{A}$ of vertices in $K_{n}$ is independent in $G(n, p)$ if no two vertices from $\mathscr{A}$ are adjacent in $G(n, p)$. The independence number $\alpha(G(n, p))$ is the size of the largest independent set. We write $\alpha_{n}(p)$ for $\alpha(G(n, p))$ below. 
For a fixed $p, \alpha_{n}(p)$ is remarkably concentrated; there exists $k=k(n, p)$ such that $\alpha_{n}(p)=k$ or $k+1$ with high probability. See Shamir and Spencer (1987) for details. In this case, our method does not produce any new result.

For $p=s / n$ with $s>0$ fixed, Bollobás and Thomason (1985) made very careful analysis of $\alpha_{n}(s / n)$. Let

$$
f(s)=\sup \left\{\beta>0: \lim _{n \rightarrow \infty} P\left(\alpha_{n}\left(\frac{s}{n}\right)>\beta n\right)=1\right\} .
$$

In the range $0<s \leqslant 1$, there is an explicit formula for $f(s)$. Although the formula is far from being pretty, it does enable one to compute particular values of $f(s)$. In the range $s>1$, there is a rather weak low bound for $f(s) ; f(s) \geqslant(s \log s-s+1) /(s-1)^{2}$. These results directly imply that in sparse random graphs the size of the largest independent sets is proportional to $n$, the size of the graph. So, it is natural to believe that the limit of the rate $E \alpha_{n}(s / n) / n$ exists. However, no rigorous proof is available. Frieze (1990) proved that by a large deviation inequality of Talagrand-type, for any fixed $\varepsilon>0$ and for sufficiently large $n \geqslant n_{0}(\varepsilon)$ and $s \geqslant s_{o}(\varepsilon)$

$$
\left|\alpha_{n}\left(\frac{s}{n}\right)-\gamma(s) n\right| \leqslant \frac{\varepsilon n}{s}
$$

with high probability, and moreover

$$
\left|E \alpha_{n}\left(\frac{s}{n}\right)-\gamma(s) n\right| \leqslant \frac{\varepsilon n}{s},
$$

where

$$
\gamma(s)=\frac{2}{s}(\log s-\log \log s-\log 2+1) .
$$

However, his method cannot provide an explicit concentration inequality for the independence number. Here we apply Theorem 1 to obtain the following.

Theorem 4. Let $\alpha_{n}=\alpha_{n}(s / n)$. Then, for any fixed $\varepsilon>0$ and for all sufficiently large $n \geqslant n_{1}(\varepsilon)$ and $s \geqslant s_{1}(\varepsilon)$

$$
P\left(\left|\alpha_{n}-E \alpha_{n}\right| \geqslant t\right) \leqslant 2 \exp \left(-\frac{t^{2}}{4(\varepsilon+(1-\varepsilon)(\gamma(\varepsilon s)+1 / s)) n+2 t / 3}\right) .
$$

Remark. For large $s$ this provides a much better tail bound than the bound obtained by simply applying Azuma's inequality. Boucheron et al. (2000) also obtained similar bounds for $\alpha_{n}$ by using the log-Sobolev inequality.

Proof. We use the vertex-expose martingale argument. First, we name the vertices in $K_{n}$ by $k, 1 \leqslant$ $k \leqslant n$. Let $U_{i j}, 1 \leqslant i<j \leqslant m, m=\left(\begin{array}{c}n \\ 2\end{array}\right)$, be the uniform weight assigned to the edge between $i$ and $j$ and let $\left\{U_{i j}^{\prime}\right\}$ be an independent copy of $\left\{U_{i j}\right\}$. For each $1 \leqslant k \leqslant n$, let $G_{k}^{\prime}(n, s / n)$ be the random graph generated by replacing the weight $U$ adjacent to the vertex $k$ by $U^{\prime}$, and write $\alpha\left(1, \ldots, k-1, k^{\prime}, k+1, \ldots, n\right)$ for the corresponding independence number. Then, we let

$$
\Delta_{k}=\alpha(1, \ldots, k-1, k, k+1, \ldots, n)-\alpha\left(1, \ldots, k-1, k^{\prime}, k+1, \ldots, n\right) .
$$


It is easy to see that $\Delta_{k}$ takes only $+1,0$, and -1 . Moreover, since $E\left(\Delta_{k} \mid \mathscr{F}_{k-1}\right)=0$ where $\mathscr{F}_{k}=$ $\sigma\left(U_{i j}, 1 \leqslant i<j \leqslant k\right)$, we have

$$
P\left(\Delta_{k}=+1 \mid \mathscr{F}_{k-1}\right)=P\left(\Delta_{k}=-1 \mid \mathscr{F}_{k-1}\right) .
$$

Denote by $A_{k}$ the event that any of the largest independent sets of $G(n, s / n)$ contains the vertex $k$. By symmetry, given $U_{i j}, 1 \leqslant i<j \leqslant k-1$, each $A_{j}, k \leqslant j \leqslant n$, occurs equally likely. More precisely, for $k \leqslant j \leqslant n$

$$
P\left(A_{k} \mid \mathscr{F}_{k-1}\right)=P\left(A_{j} \mid \mathscr{F}_{k-1}\right) .
$$

Thus, we have

$$
\begin{aligned}
P\left(\Delta_{k}=+1 \mid \mathscr{F}_{k-1}\right) & \leqslant P\left(A_{k} \mid \mathscr{F}_{k-1}\right) \\
& =\frac{1}{n-k+1} \sum_{j=k}^{n} P\left(A_{j} \mid \mathscr{F}_{k-1}\right) \\
& =\frac{1}{n-k+1} E\left(\sum_{j=k}^{n} 1\left(A_{j}\right) \mid \mathscr{F}_{k-1}\right) .
\end{aligned}
$$

Note that $\sum_{j=k}^{n} 1\left(A_{j}\right)$ is just the number of vertices which are common to any largest independent sets of $G(n, s / n)$ and hence if we collect the vertices $j$ with $1\left(A_{j}\right)=1$, this forms an independent set of the random graph on $\{k, k+1, \ldots, n\}$ where each edge appears with probability $s / n$. Therefore, by the definition of the independent set we have

$$
E\left(\sum_{j=k}^{n} 1\left(A_{j}\right) \mid \mathscr{F}_{k-1}\right) \leqslant E \alpha_{n-k+1}\left(\frac{s}{n}\right) \quad \text { a.s. }
$$

Now a direct application of Theorem 1 gives

$$
P\left(\left|\alpha_{n}-E \alpha_{n}\right| \geqslant t\right) \leqslant 2 \exp \left(-\frac{t^{2}}{4 \sum_{k=1}^{n} E \alpha_{k}(s / n) / k+2 t / 3}\right) .
$$

Given $\varepsilon>0$, in view of (3.2), for $k \geqslant n_{0}(1), \varepsilon s \geqslant s_{0}(1)$ we have

$$
\left.\mid E \alpha_{k}\left(\frac{\varepsilon s}{k}\right)\right)-\gamma(\varepsilon s) k \mid \leqslant \frac{k}{s} \text {. }
$$

Thus, since $\alpha_{k}(s / n) \leqslant k$, it easily follows that for all sufficiently large $n$ and $s$

$$
\begin{aligned}
\sum_{k=1}^{n} \frac{E \alpha_{k}\left(\frac{s}{n}\right)}{k} & =\sum_{k \leqslant \varepsilon n} \frac{E \alpha_{k}(s / n)}{k}+\sum_{k>\varepsilon n} \frac{E \alpha_{k}(s / n)}{k} \\
& \leqslant \varepsilon n+\sum_{k>\varepsilon n} \frac{E \alpha_{k}(\varepsilon s / k)}{k} \\
& \leqslant \varepsilon n+(1-\varepsilon)\left(\gamma(\varepsilon s)+\frac{1}{s}\right) n .
\end{aligned}
$$

Now the theorem follows from (3.3). 


\section{References}

Aldous, D.J., Diaconis, P., 1999. Longest increasing subsequences: From patience sorting to the Baik-Deift-Johansson theorem. Bull. Amer. Math. Soc. 36, 413-432.

Azuma, K., 1967. Weighted sums of certain dependent variables. Tôhoku Math. J. 3, 357-367.

Baik, J., Deift, P., Johansson, K., 1999. On the distribution of the length of the longest increasing subsequence of random permutations. J. Amer. Math. Soc. 12, 1119-1178.

Bollobás, B., Brightwell, G., 1992. The height of a random partial order: concentration of measure. Ann. Appl. Probab. 2, 1009-1018.

Bollobás, B., Thomason, A.G., 1985. Random graphs of small order. In: Karoński, M., Ruciński, A. (Eds.), Random graphs'83, Proceedings, Poznań, 1983. North-Holland, Amsterdam, New York, pp. 47-97.

Bollobás, B., Winkler, P.M., 1988. The longest chain among random points in Euclidean space. Proc. Amer. Math. Soc. 103, 347-353.

Boucheron, S., Lugosi, G., Massart, P., 2000. A sharp concentration inequality with applications. Random Struct. Alg. 16, $277-292$.

Deift, P., 2000. Integrable systems and combinatorical theory. Notices AMS. 47, 631-640.

Frieze, A.M., 1990. On the independence number of random graphs. Discrete Math. 81, 171-175.

Godbole, A., Hitczenko, P., 1998. Beyond the method of bounded differences. DIAMCS ser., Discrete Mathematics and Theoretical Computer Science 41, 43-57.

Hammersley, J.M., 1972. A few seedlings of research. Proceedings of the Sixth Berkeley Symposium on Mathematical and Statistical Probability, University of California Press, Berkeley, CA, pp. 345-394.

Hoeffding, W., 1963. Probability inequalities for sums of bounded random variables. J. Amer. Statist. Assoc. 58, 13-20.

Janson, S., Luczak, T., Rucinski, A., 2000. Random Graphs. Wiley, New York.

Luczak, M.J., McDiarmid, C., 2001. Bisecting sparse random graphs. Random Struct. Algebra 18, 31-38.

McDiarmid, C., 1989. On the Method of Bounded Differences. Surveys in Combinatorics. Cambridge University Press, Cambridge, pp. 148-188.

McDiarmid, C., 1997. Concentration. In: Habib, M., McDiarmid, C., Ramirez-Alfonsin, J., Reed, B. (Eds.), Probabilistic methods for algorithmic discrete mathematics. Springer, New York, pp. 195-248.

Shamir, E., Spencer, J., 1987. Sharp concentration of chromatic numbers on random graphs $G_{n, p}$. Combinatorica 7 , 124-129.

Steele, J.M., 1995. Variations on the long increasing subsequence theme of Erdős and Szekeres. In: Aldous, D., Diaconis, P., Steele, J.M. (Eds.), Discrete probability and algorithms, Volumes in Mathematics and its applications, Vol. 72. Springer, New York, pp. 111-131.

Steele, J.M., 1997. Probability Theory and Combinatorial Optimization. SIAM, Philadelphia.

Talagrand, M., 1995. Concentration of measure and isoperimetric inequalities in product spaces. Publ. Math. del's IHES $81,73-205$.

$\mathrm{Vu}$, V.H., 2001. Concentration of non-Lipschitz functions and applications, preprint. 